\title{
CORRECTED RMS ERROR AND EFFECTIVE NUMBER OF BITS FOR SINEWAVE ADC TESTS
}

\author{
Jerome J. Blair \\ Bechtel Nevada, Las Vegas, Nevada, USA \\ Phone: 702/295-2647, Fax: 702/295-3305 email: blairjj@nv.doe.gov \\ Thomas E Linnenbrink \\ Q-DOT, Inc., A Simtek Company, Colorado Springs, Colorado, USA \\ Phone: 719/590-1112, Fax: 719/590-1125, email: toml@qdot.com
}

\begin{abstract}
A new definition is proposed for the effective number of bits of an ADC. This definition removes the variation in the calculated effective bits when the amplitude and offset of the sinewave test signal is slightly varied. This variation is most pronounced when test signals with amplitudes of a small number of code bin widths are applied to very low noise ADC's. The effectiveness of the proposed definition is compared with that of other proposed definitions over a range of signal amplitudes and noise levels.
\end{abstract}

Keywords - ADC, Analog-to-Digital Converter, Waveform Recorder, Effective Number of Bits, Noise.

\section{INTRODUCTION}

A fundamental technique for assessing the accuracy of analog to digital converters (ADC's) is to apply a sinewave signal to the input of the device under test, calculate the rms deviation, $e_{r m s}$, between a sinewave fitted to the data and the data itself, and calculate other performance measures, such as signal-to-noise-and-distortion (SINAD) ratio and effective number of bits from the rms deviation. An important element of the performance evaluation involves the comparison of the rms deviation with its ideal value.

The two IEEE standards (i.e., IEEE Stds 1057-1994 and 1241-2000) that cover such evaluations [1,2] assume that the ideal rms deviation is given by $Q / \sqrt{12}$, where $Q$ is the average code bin width of the ADC. It is well known $[3,4]$ that for sinewave inputs this is only an approximation and that the approximation error becomes noticeable if the ADC has six or less bits or if the amplitude of the input signal is very small.

The definition for effective number of bits $(E N O B)$ in the IEEE standards is given by
$E N O B=\log _{2}\left(\frac{F E_{I r m s}}{Q e_{r m s}}\right)=\log _{2}\left(\frac{F}{\sqrt{12} e_{r m s}}\right)$,

where $F$ is the full-scale range of the $\mathrm{ADC}, Q$ is the average code bin width and $E_{\text {Irms }}=Q / \cdot \sqrt{12}$ is the nominal ideal rms error. The nominal ideal rms error is based on the assumption that the quantization error has a uniform distribution, while for a sinewave the quantization error will be a value $e_{\text {Irms }}$, which will depend on the offset and amplitude of the sinewave input signal. The calculated $E N O B$ for an ideal ADC will then be

$E N O B_{\text {Ideal }}=\log _{2}\left(\frac{F E_{\text {Irms }}}{Q e_{\text {Irms }}}\right)=N-\log _{2}\left(\frac{e_{\text {Irms }}}{E_{\text {Irms }}}\right)$,

where $N$ is the number of bits in the ADC. Thus, for an ideal $\mathrm{ADC}$, one in which the only error is quantization error, the calculated $E N O B$ will vary with small changes in the input signal--giving a misleading indication of ADC performance.

To remedy this situation Hejn, Pacut and Kramarski (HPK) [4] proposed the new definition of effective bits given by

$E N O B_{H P K}=\log _{2}\left(\frac{F e_{\text {Irms }}}{Q e_{r m s}}\right)$,

in which $E_{\text {Irms }}$ has been replaced by $e_{\text {Irms. }}$ When testing an ideal $\mathrm{ADC}$ we obtain $E N O B_{H P K}=N$, as desired. However, if the error is dominated by sources other than quantization error, as is the case for most commercial ADC's, the IEEE standard definition of $E N O B$ will give a result that is independent of small changes in signal amplitude and offset, while $E N O B_{H P K}$ varies substantially with the signal. 
We propose a new definition of $E N O B$, called the standardized effective number of bits -- $E N O B_{S}$, which also produces the correct value for an ideal ADC for any sinewave offset and amplitude and gives more accurate results than the HPK method with moderate noise. We give simple procedures for calculating $E N O B_{S}$ and compare the results of the three definitions in a meaningful variety of situations.

\section{Variation of IEEE Standard ENOB with signal amplitude and offset}

We consider an ideal ADC with no errors other than quantization error. In this situation we would want the number of effective bits to be the actual number of bits of the ADC, independent of the input signal. The rms error for a sinewave signal deviates from the ideal value of $Q / \sqrt{12}$, because the distribution of errors when sampling a sinewave differs from a uniform distribution. Sinewaves are sampled more frequently near the peaks than near the zero crossings. Hence, sinewaves with peaks near the centers of code bins (where the error is minimum) will have a smaller rms error than the ideal, while sinewaves with peaks near the edges of code bins (where the error is maximum) will have a larger rms error than the ideal.

This variation in rms error with small changes in sinewave offset and amplitude leads to a change in the number of effective bits. We wish to quantify the variation in the number of effective bits due to this phenomenon. For a signal with amplitude $a$ and offset $d$, let

$$
\begin{aligned}
& E N O B_{\max }(a, d)= \\
& N-\min \left\{\log _{2}\left(\frac{e_{\text {Irms }}\left(a^{\prime}, d^{\prime}\right)}{E_{\text {Irms }}}\right): \begin{array}{l}
a-Q / 2 \leq a^{\prime} \leq a+Q / 2 \\
d-Q / 2 \leq d^{\prime} \leq d+Q / 2
\end{array}\right\},
\end{aligned}
$$

where $e_{\text {Irms }}(a, d)$ is the ideal rms error for signals with amplitude $a$ and offset $d$. The expression $\min \{x: y\}$ means the minimum value of $\mathrm{x}$ subject to the constraints given in $\mathrm{y} ;$ an analogous meaning applies to $\max \{x: y\}$. The quantity, $E N O B_{\max }$ is the maximum number of effective bits for any signal with amplitude and offset near $a$ and $d$. We similarly define

$$
\begin{aligned}
& E N O B_{\min }(a, d)= \\
& \left.N-\max \left\{\log _{2}\left(\frac{e_{\text {Irms }}\left(a^{\prime}, d^{\prime}\right)}{E_{\text {Irms }}}\right)\right) \begin{array}{l}
a-Q / 2 \leq a^{\prime} \leq a+Q / 2 \\
d-Q / 2 \leq d^{\prime} \leq d+Q / 2
\end{array}\right\} .
\end{aligned}
$$

We have found that the maximum and minimum always occur at two different values for the offset and that the maximum and minimum at these two values of offset are always the same. The two values for offset are: the value that puts the zero crossing of the sinewave at the center of a code bin, and the value that puts the zero crossing of the sinewave at the edge of a code bin. This is not surprising, because with these two offsets the positive and negative peaks of the sinewave have their maximum (or minimum) error for the same amplitude. In our evaluations we have fixed the offset so that the zero crossing of the sinewave is at the center of a code bin and varied only the amplitude.

Note that in (4) and (5) the difference between the maximum and minimum $E N O B$ and the actual number of bits is independent of $N$, the number of bits. It depends only on the amplitude of the signal in code bin widths. Figure 1 shows the error in the $E N O B$ as a function of the signal amplitude. For small signals this is noticeable but, in most applications, not significant. A change of 0.1 in the value of $E N O B$ corresponds to a seven per cent deviation of the rms error from its nominal value.

Each figure has two parts. The first part shows the error for signal amplitudes less than 32 code bin widths. The second part shows the error for amplitudes up to 128 code bin widths (256 peak-to-peak.) The second part is shown with a compressed scale to better display the results at large amplitudes. The first part is shown at a scale that includes the larger error at an amplitude of one code bin width.

Details of how the calculations were performed and the reason for the spread in $E N O B$ for large amplitudes (near 128) will be covered later.

\section{Variation of HPK ENOB with signal amplitude}

For an ideal ADC there will be no error in the ENOB calculated by the HPK method. The denominator in the argument of the logarithm in (4) and (5) is replaced with $\mathrm{e}_{\text {Irms }}$ with this definition, making the logarithm zero. However, if there are errors other than the quantization error the situation is different.

Figure 2 shows the error in the HPK ENOB when random noise has been added. The amount of random noise is $\sqrt{15 / 12} Q$, the amount sufficient to reduce the number of effective bits by two. This is a reasonable amount of noise for a commercial high-speed ADC. The error curves in Figure 2 are almost identical to those in Figure 1. Thus, the errors for the HPK definition are essentially the same as those for the IEEE standard definition, but they occur in a different situation. 

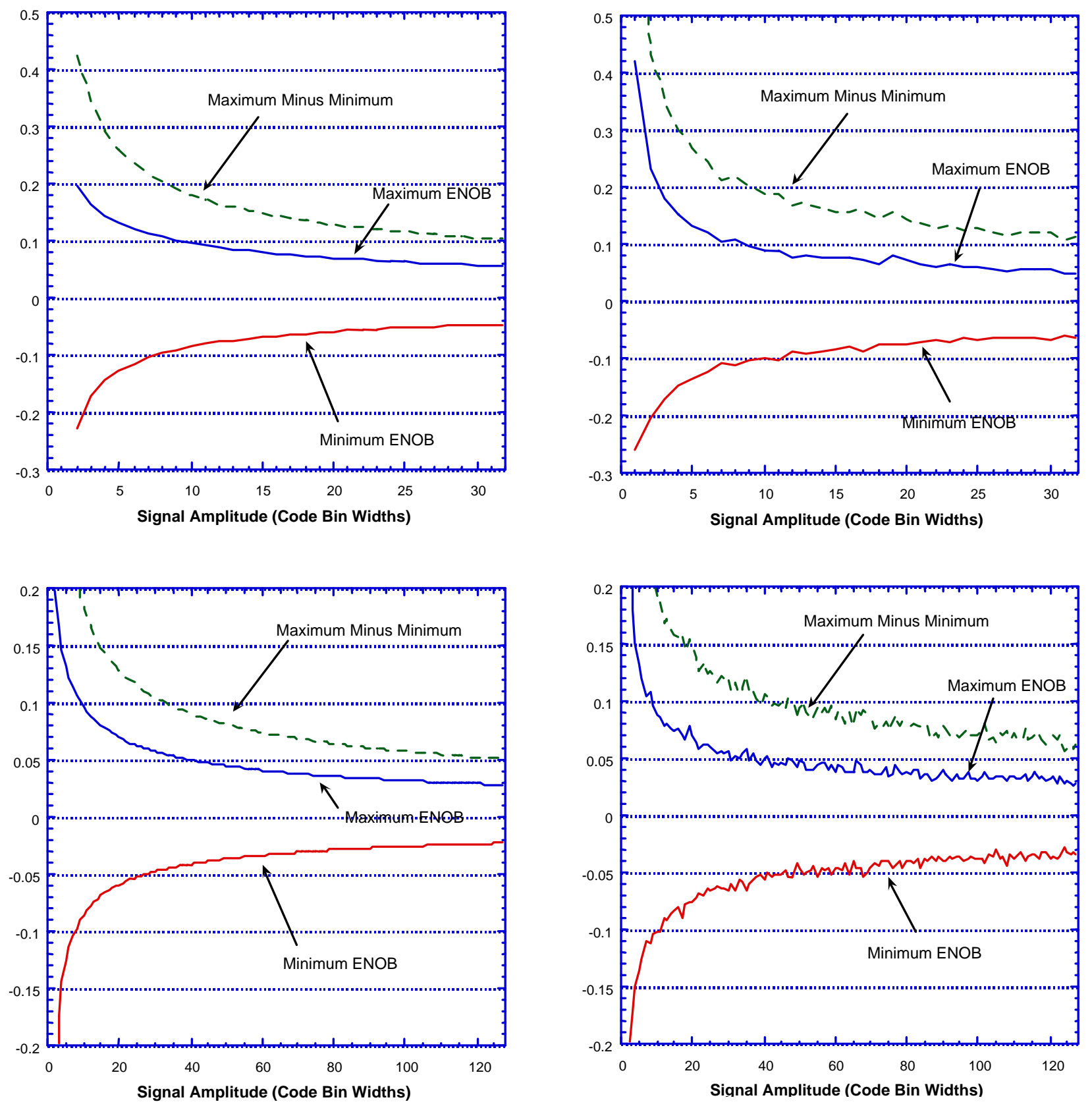

Figure 1. The minimum and maximum $E N O B$ (relative to the actual ENOB) and the difference between the two as a function of signal amplitude. The minimum and maximum are calculated for all signal amplitudes within one-half code bin width of the value specified on the horizontal axis.

Figure 2. The minimum and maximum ENOB (relative to the actual ENOB, using the HPK definition) and the difference between the two as a function of signal amplitude. The minimum and maximum are calculated for all signal amplitudes within one-half code bin width of the value specified on the horizontal axis. Results are for an $A D C$ with sufficient noise to reduce the ENOB by two. 


\section{PROPOSED NEW DEFINITION FOR ENOB}

We want a definition of $E N O B$ that gives the expected answer and depends only slightly on the amplitude and offset of the signal. For both of the previously discussed definitions the error in $E N O B$ was caused by the difference between $e_{\text {Irms, }}$ the ideal rms error for the specific signal, and $E_{\text {Irms }}$, the ideal rms error under the assumption of uniformly distributed error. To take this difference into account we introduce the quantity

$e_{C r m s}=\sqrt{e_{r m s}^{2}-e_{I r m s}^{2}}$,

the corrected rms error. This is an estimate of the rms error that is in addition to the quantization error. This estimate is valid if the additional error is uncorrelated with the quantization error, a reasonable assumption. We next introduce the standardized rms error given by

$e_{\text {Srms }}=\sqrt{e_{\text {Crms }}^{2}+Q^{2} / 12}=\sqrt{e_{\text {rms }}^{2}-e_{\text {Irms }}^{2}+Q^{2} / 12}$.

This is an estimate of the rms error under the condition of uniformly distributed quantization error. We define the standardized effective number of bits, $E N O B_{\mathrm{S}}$, by replacing $e_{r m s}$ in (1) with $e_{S r m s}$. Here are several equivalent expressions for this.

$$
\begin{aligned}
& E N O B_{S}=\log _{2}\left(\frac{F E_{\text {Irms }}}{Q e_{\text {Srms }}}\right)=\log _{2}\left(\frac{F}{\sqrt{12} e_{\text {Srms }}}\right)= \\
& N-\log _{2}\left(\frac{e_{\text {Srms }}}{E_{\text {Irms }}}\right)=N-\frac{1}{2} \log _{2}\left(\frac{e_{r m s}^{2}+E_{\text {Irms }}^{2}-e_{\text {Irms }}^{2}}{E_{\text {Irms }}^{2}}\right)
\end{aligned}
$$

For an ideal ADC, one for which $e_{r m s}^{2}=e_{\text {Irms }}^{2}$, we can see from the last expression in (8) $E N O B_{S}=N$, independent of the input signal. The formula reduces to the same one as the HPK definition in this case. For the situation in which random noise dominates, the term $E_{\text {Irms }}^{2}-e_{\text {Irms }}^{2}$ can be neglected compared to $e_{r m s}^{2}$, so the expression reduces to that in the IEEE standard definition. The situation for intermediate cases will be established by simulation.

\section{Calculation of $\mathbf{e}_{\text {Irms }}$}

Use of the proposed definition of $E N O B$ requires calculation of $e_{\text {Irms }}$, the ideal rms error for the particular amplitude and offset of the sinewave used for the measurement. Reference [4] gives a complicated approach to calculate an approximation. We give a simple approach with fewer sources of error.
The approach given here assumes that the input data to the sine-fit procedure is the integer output of the ADC. Let $f(t)$ be the fitted sinewave, obtained by any appropriate method. Then the estimate is given by

$e_{\text {Irms }}^{2}=\frac{1}{M} \sum_{i=1}^{M}\left(f\left(t_{i}\right)-\operatorname{round}\left(f\left(t_{i}\right)\right)^{2}\right.$,

where round is the function that rounds to the nearest integer, and $M$ is the number of samples in the record. This is the estimate used for the simulations.

\section{COMPARISON RESULTS}

\section{Simulation Computations}

All simulations used one cycle of a sinewave sampled at 32768 uniformly spaced times. The ENOB was calculated using the 3-parameter sine fit [1,2]. An ADC with a $Q$ of 1 was used, giving integer signal output values. The results for error in $E N O B$ are given for integer values of sinewave amplitude (peak amplitudes, not peakto-peak.) For each integer value, $a$, of sinewave amplitude, 51 simulations were performed for amplitudes between $a-1 / 2$ and $a+1 / 2$ with an interval between amplitudes of 0.02. For each of the simulations the $E N O B$ was calculated. The results shown in the fugures which follow are the differences between the maximum and the minimum of these 51 values of $E N O B$.

Random noise was added to the signals before quantization. The noise was zero mean normally distributed white noise. Three different noise standard deviations were used: $\sigma=1 / \sqrt{12}$, giving a loss of onehalf effective bit; $\sigma=1 / 2$, giving a loss of one effective bit; and $\sigma=\sqrt{15 / 12}$, giving a loss of two effective bits. The statement that a quantity of random noise gives a loss of $p$ effective bits means that an ideal ADC with $N$ bits and that quantity of random noise will have an $E N O B$ if $N$ $-p$.

A different random noise sequence was used for each of the 51 simulations for each amplitude. Thus the variation in $E N O B$ will contain the statistical fluctuations. The expected size of these fluctuations will be discussed next.

If the main contribution to the error is normally distributed random noise, the $E N O B$ will be a random variable with standard deviation,

$$
\sigma_{E}=\frac{1}{\ln (2) \cdot \sqrt{2 M}} \cong \frac{1.02}{\sqrt{M}},
$$


where $M$ is the number of samples in a record. The value of this is 0.0056 for the record size we have used. The expected value for the maximum minus the minimum of 51 values is $4.4 \sigma_{E}=0.025$. This is the minimum variation we would expect to observe in the $E N O B$.

\section{Description of Figures}

Figure 3 shows the variation in $E N O B$ using the IEEE standard definition. The upper curve is for an ideal ADC (zero lost bits). The next curve below is for one-half lost bit. The curves for one and two lost bits lie on top of each other and are at the level expected due to statistical variation alone.

Figure 4 shows the results for the HPK definition. The two overlaying curves are for one and two lost bits. The next curve down is for one-half lost bit. The curve for zero lost bits would be identically zero and is not shown. The curve for one-half lost bit is slightly worse than the corresponding curve for the IEEE standard definition.

Figure 5 shows the results for the proposed definition. The highest curve is for one lost bit. The next curve down is for one-half lost bit, and the lowest curve is for two lost bits. The curve for zero lost bits would be identically zero and is not shown. The data shows that the proposed definition is uniformly better than the HPK definition. For one or more lost bits, the IEEE standard definition is clearly the best. For one-half lost bit the proposed definition performs slightly better than the IEEE standard definition.

The net result of this is that for an ADC with one-half or more lost bits the IEEE standard definition gives the most accurate results. For low-noise ADC's (less than one-half lost bits) the proposed definition gives the most repeatable results with respect to variations in signal amplitude and offset.

\section{CONCLUSIONS}

A modified definition of $E N O B$ was presented which partially solves the problem of the calculated $E N O B$ being sensitive to small changes in the sinewave amplitude and offset. The accuracy of the proposed definition was compared to other definitions. It was found that for ADC's with one-half or more lost bits due to noise, the IEEE standard definition performs best. For low noise ADC's, those with one-half or less lost bits, the proposed definition works best. The HPK method gives the same result as the proposed method for the noise-free case, but its performance diminishes as noise is added.
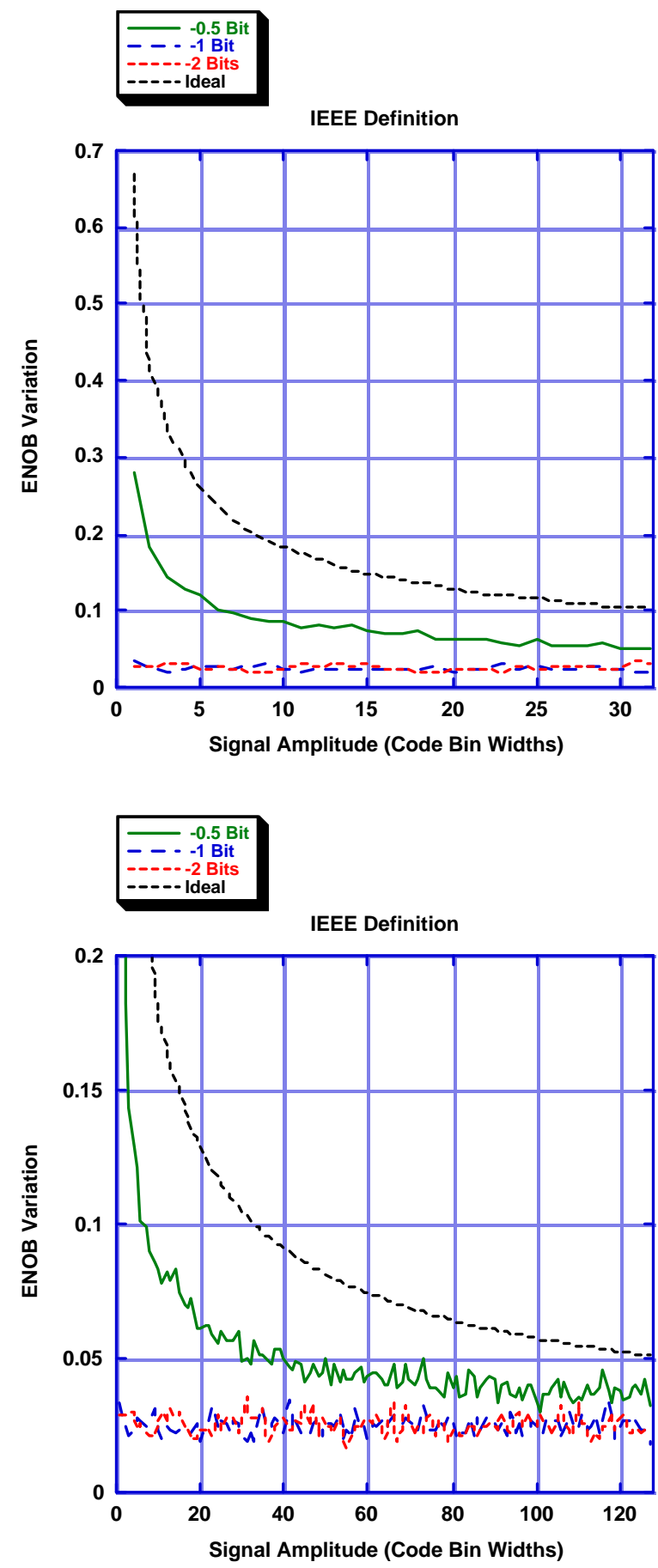

Figure 3. The variation in ENOB using the IEEE standard definition. The upper curve is for an ideal ADC (zero lost bits). The next curve below is for one-half lost bit. The curves for one and two lost bits lie on top of each other and are at the level expected due to statistical variation alone. 

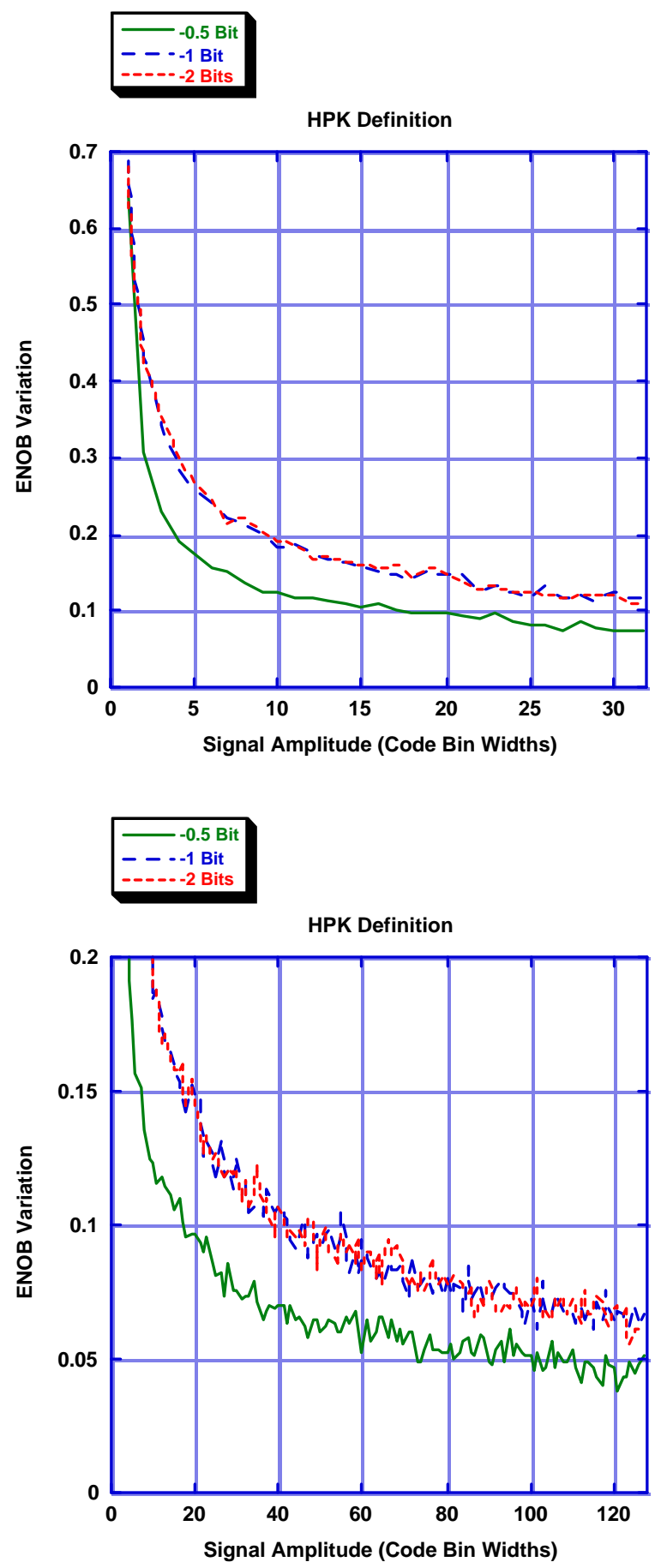

Figure 4. Results for the HPK definition. The two overlaying curves are for one and two lost bits. The next curve down is for one-half lost bit. The curve for zero lost bits would be identically zero and is not shown. The curve for one-half lost bit is slightly worse than the corresponding curve for the IEEE standard definition.
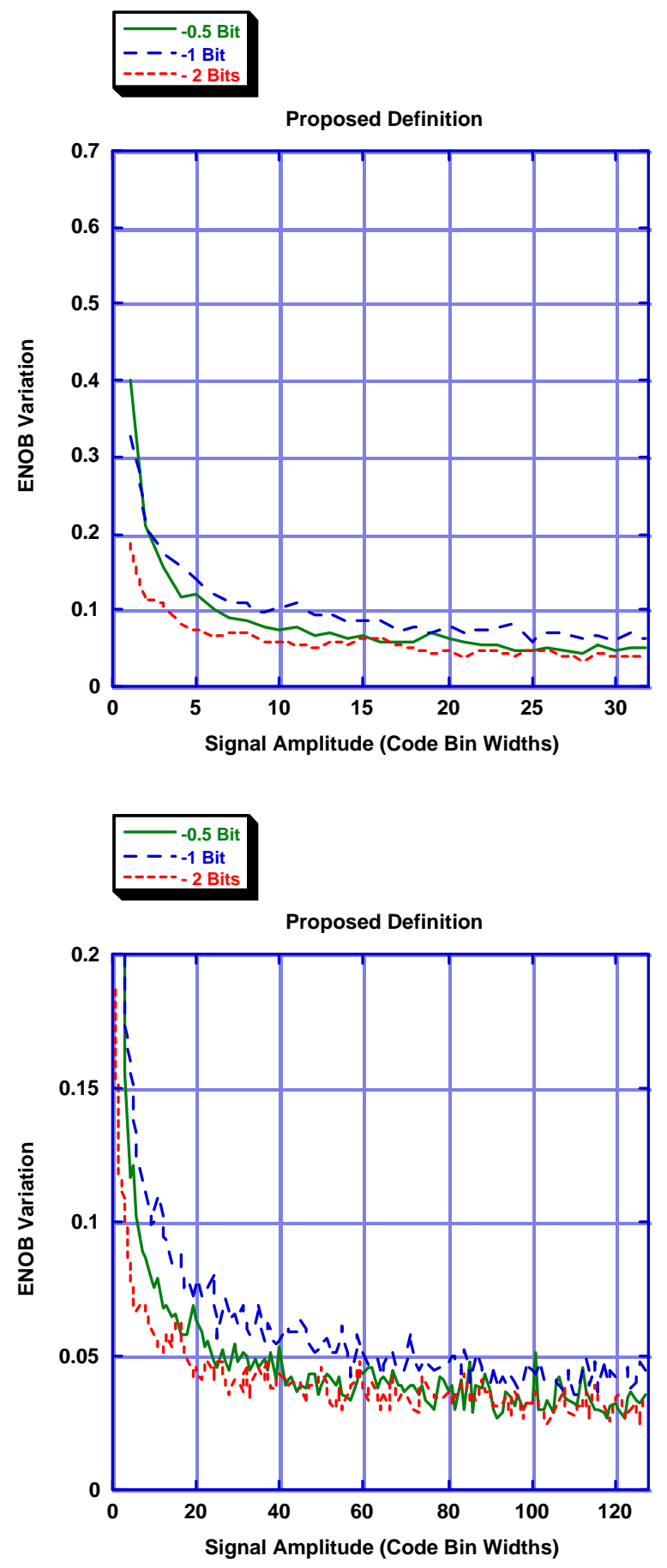

Figure 5. Results for the proposed definition. The highest curve is for one lost bit. The next curve down is for onehalf lost bit, and the lowest curve is for two lost bits. The curve for zero lost bits would be identically zero and is not shown. 


\section{REFERENCES}

[1] IEEE Standard 1057-1994, "IEEE Standard for Digitizing Waveform Recorders."

[2] IEEE Standard 1241-2000, "IEEE Standard for Terminology and Test Methods for Analog-to-Digital Converters."

[3] M. F. Wagdy and W. M. Ng, "Validity of uniform quantization error model for sinusoidal signals without and with dither," IEEE Trans. Instrum. Measur., vol. 38, pp. 718-722, June 1989.

[4] Hejn, K., Pacut, A. and Kramarski, L., "The effective resolution measurements in scope of sine-fit test," IEEE Trans. Instrum. Measur., vol. 47, pp. 45-50, Feb. 1998.

DOE/NV11718--672. This work was supported by the U. S. Department of Energy, National Nuclear Security Administration, Nevada Operations Office, under contract No. DE-AC08-96NV1178.
By acceptance of this article, the publisher and/or recipient acknowledges the U. S. Government's right to retain a nonexclusive, royalty-free license in and to any copyright covering this paper.

This product was prepared as an account of work sponsored by an agency of the United States Government. Neither the United States Government nor any agency thereof, nor any of their employees, nor any of their contractors, subcontractors or their employees, makes any warranty, express or implied, or assumes any legal liability or responsibility for the accuracy, completeness, or any third party's use or the results of such use of any information, apparatus, product, or process disclosed, or represents that its use would not infringe privately owned rights. Reference herein to any specific commercial product, process, or service by trade name, trademark, manufacturer, or otherwise, does not necessarily constitute or imply its endorsement, recommendation, or favoring by the United States Government or any agency thereof or its contractors or subcontractors. The views and opinions of authors expressed herein do not necessarily state or reflect those of the United States Government or any agency thereof. 